\title{
Convergence Analysis of General Version of Gauss-Type Proximal Point Method for Metrically Regular Mappings
}

\author{
Md. Asraful Alom ${ }^{1,2}$, Mohammed Harunor Rashid1, Kalyan Kumer Dey ${ }^{1}$ \\ ${ }^{1}$ Department of Mathematics, University of Rajshahi, Rajshahi, Bangladesh \\ ${ }^{2}$ Department of Mathematics, Faculty of Civil Engineering, Khulna University of Engineering \& Technology, \\ Khulna, Bangladesh \\ Email: asraf.math.kuet@gmail.com,harun_math@ru.ac.bd,kkdmath@yahoo.com
}

Received 7 January 2016; accepted 17 July 2016; published 20 July 2016

Copyright (C) 2016 by authors and Scientific Research Publishing Inc.

This work is licensed under the Creative Commons Attribution International License (CC BY).

http://creativecommons.org/licenses/by/4.0/

(c) (i) Open Access

\section{Abstract}

We introduce and study in the present paper the general version of Gauss-type proximal point algorithm (in short GG-PPA) for solving the inclusion $0 \in T(x)$, where $T$ is a set-valued mapping which is not necessarily monotone acting from a Banach space $X$ to a subset of a Banach space $Y$ with locally closed graph. The convergence of the GG-PPA is present here by choosing a sequence of functions $g_{k}: X \rightarrow Y$ with $g_{k}(0)=0$, which is Lipschitz continuous in a neighbourhood $O$ of the origin and when $T$ is metrically regular. More precisely, semi-local and local convergence of GG-PPA are analyzed. Moreover, we present a numerical example to validate the convergence result of GG-PPA.

\section{Keywords}

Set-Valued Mappings, Metrically Regular Mappings, Lipschitz-Like Mapping, Local and Semi-Local Convergence

\section{Introduction}

We are concerned in this study with the problem of finding a point $x \in \Omega \subseteq X$ satisfying

$$
0 \in T(x)
$$

where $T: X \rightrightarrows 2^{Y}$ is a set-valued mapping and $X$ and $Y$ are Banach spaces. This type of inclusion is an abstract 
model for a wide variety of variational problems including complementary problems, system of nonlinear equations and variational inequalities. In particular, it may characterize optimality or equilibrium problems. Choose a sequence of functions $g_{k}: X \rightarrow Y$ with $g_{k}(0)=0$ which is Lipschitz continuous in a neighborhood $O$ of the origin.

Martinet [1] proposed the following algorithm for the first time for applying it to convex optimization by considering a sequence of scalars $\left\{\lambda_{k}\right\}$, which are different from zero:

$$
0 \in \lambda_{k}\left(x_{k+1}-x_{k}\right)+T\left(x_{k+1}\right) \text {, for each } k=0,1,2, \cdots
$$

Rockafellar [2] thoroughly explored the method (2) in the general framework of maximal monotone inclusions. In particular, Rockafellar ([2], Theorem 1) shows that when $x_{k+1}$ is an approximate solution of (2) and $T$ is maximal monotone, then for a sequence of positive scalars $\lambda_{k}$ the iteration (2) generates a sequence $\left\{x_{k}\right\}$ which is weakly convergent to a solution of (1) for any starting point $x_{0} \in X$. In [3], Aragón Artacho et al. have been presented the general version of the proximal point algorithm (GPPA) (see Algorithm 1), for the case of nonmonotone mappings, for solving the inclusion (1).

Let $x \in X$. The subset of $X$, denoted by $D^{k}(x)$, is defined by

$$
D^{k}(x):=\left\{d \in X: 0 \in g_{k}(d)+T(x+d)\right\} .
$$

Thus we have the following algorithms which have been presented by Aragón Artacho et al. [3]:

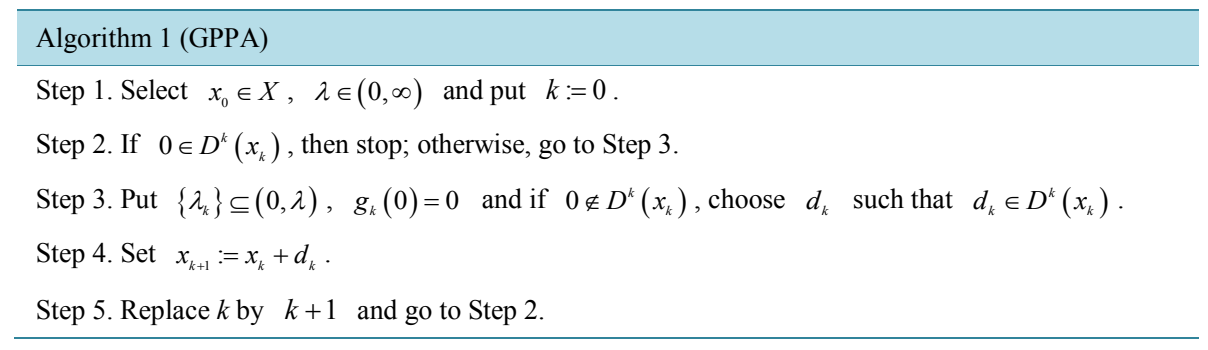

Note that, for a starting point near to a solution, the sequences generated by Algorithm 1 are not uniquely defined and not every sequence is convergent. The results obtained in [3] guarantee the existence of one sequence, which is convergent. Therefore, from the viewpoint of numerical computation, we can assume that these kinds of methods are not suitable in practical application. This drawback motivates us to introduce a method "socalled" general version of Gauss-type proximal point algorithm (GG-PPA). The difference between Algorithm 1 and our proposed Algorithm 2 is that the GG-PPA generates sequences, whose every sequence is convergent, but this does not happen for Algorithm 1. Thus we propose here the GG-PPA as follows:

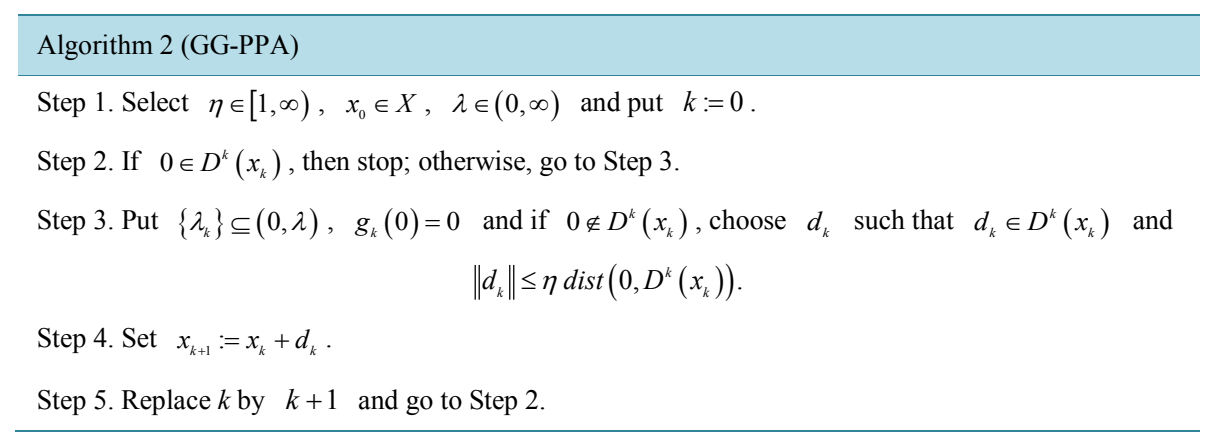

We observe, from Algorithm 2, that

1) if $g_{k}(u)=\lambda_{k} u$ and then we assume $Y=X$ a Hilbert space, this algorithm reduces to the classical proximal point algorithm defined by (2).

2) if $g_{k}(u)=\lambda_{k} u$, Algorithm 2 is equivalent to the classical Gauss-type proximal point method, which has been introduced by Rashid et al. [4]. 
A large number of authors have been studied on proximal point algorithm and have also found applications of this method to specific variational problems. Most of the study on this subject have been concentrated on various versions of the algorithm for solving inclusions involving monotone mappings, and specially, on monotone variational inequalities (see in [5]-[8]). Spingarn [9] has been studied first weaker form of monotonicity and for details see in [10].

There have a large study on local convergence analysis about Algorithm 1 (cf. [3] [11] [12]), but there is no semilocal analysis for Algorithm 1. A huge number of contributions have been studied on semilocal analysis for the Gauss-Newton method (cf. [4] [13]-[16]). In [4], Rashid et al. have given a semilocal convergence analysis for the classical Gauss-type proximal point method. As our best knowledge, there is no study on semilocal analysis for Algorithm 2. Therefore we conclude that the contributions presented in this study are seems new.

In the present paper, our aim is to study the semilocal convergence for the GG-PPA defined by Algorithm 2. The metric regularity property and Lipschitz-like property for set-valued mappings are mainly used in our study. The main results are convergence analysis, established in section 3, which based on the attraction region around the initial point and provide some sufficient conditions ensuring the convergence to a solution of any sequence generated by Algorithm 2. As a consequence, local convergence results for GG-PPA are obtained.

This paper is arranged as follows. In Section 2, some necessary notations, notions and preliminary results are presented. In Section 3, we consider the GG-PPA which is introduced in Section 1 and by using the concept of metric regularity property for the set valued mapping $\mathrm{T}$, we will show the existence and present the convergence of the sequence generated by Algorithm 2. In Section 4, we present a numerical experiment to validate the semilocal convergence of Algorithm 2. In the last Section, we will give a summary of the major results to close our paper.

\section{Notations and Preliminary Results}

In the whole paper, we assume that $X$ and $Y$ are Banach spaces. Let $F$ be a set-valued mapping from $X$ into the subsets of $Y$, denoted by $F: X \rightrightarrows 2^{Y}$. Let $x \in X$ and $r>0$. The closed ball centered at $x$ with radius $r$ is denoted by $\mathbb{B}_{r}(x)$. The domain $\operatorname{dom} F$, the inverse $F^{-1}$ and the graph $\operatorname{gph} F$ of $F$ are respectively defined by

$$
\begin{array}{r}
\operatorname{dom} F:=\{x \in X: F(x) \neq \varnothing\}, \\
F^{-1}(y):=\{x \in X: y \in F(x)\}
\end{array}
$$

and

$$
\operatorname{gph} F:=\{(x, y) \in X \times Y: y \in F(x)\} .
$$

All the norms are denoted by $\|\cdot\|$. Let $A \subseteq X$ and $C \subseteq X$. The distance from $x$ to $A$ is defined by

$$
\operatorname{dist}(x, A):=\inf \{\|x-a\|: a \in A\} \text { for each } x \in X,
$$

while the excess from the set $C$ to the set $A$ is defined by

$$
e(C, A):=\sup \{\operatorname{dist}(x, A): x \in C\} .
$$

From [4], we recall the following definition of metric regularity for set-valued mapping.

Definition 1 Let $F: X \rightrightarrows Y$ be a set-valued mapping and let $(\bar{x}, \bar{y}) \in \operatorname{gph} F$. Let $r_{\bar{x}}>0, r_{\bar{y}}>0$ and $\kappa>0$. Then $F$ is said to be

1) metrically regular at $(\bar{x}, \bar{y})$ on $\mathbb{B}_{r_{\bar{x}}}(\bar{x}) \times \mathbb{B}_{r_{\bar{y}}}(\bar{y})$ with constant $\kappa$ if

$$
\operatorname{dist}\left(x, F^{-1}(y)\right) \leq \kappa \operatorname{dist}(y, F(x)) \text { for all } x \in \mathbb{B}_{r_{\bar{x}}}(\bar{x}), y \in \mathbb{B}_{r_{\bar{y}}}(\bar{y}) .
$$

2) metrically regular at $(\bar{x}, \bar{y})$ if there exist constants $r_{\bar{x}}^{\prime}>0, r_{\bar{y}}^{\prime}>0$ and $\kappa>0$ such that $F$ is metrically regular at $(\bar{x}, \bar{y})$ on $\mathbb{B}_{r_{\bar{x}}}^{\prime}(\bar{x}) \times \mathbb{B}_{r_{\bar{y}}}^{\prime}(\bar{y})$ with constant $\kappa$.

The infimum of the set of values $\kappa$ for which (4) holds is the modulus of metric regularity, denoted by 
$\operatorname{reg} F(\bar{x} \mid \bar{y})$. The absence of metric regularity at $\bar{x}$ for $\bar{y}$ corresponds to $\operatorname{reg} F(\bar{x} \mid \bar{y})=\infty$. The inequality (4) has direct use in providing an estimate for how far a point $x$ is from being a solution to the generalized equation $y \in F(x)$ and the expression $\operatorname{dist}(y, F(x))$ measures the residual when $y \in F(x)$.

Recall the definition of Lipschitz-like continuity for set-valued mapping from [17]. This notion was introduced by Aubin in [18] and has been studied extensively.

Definition 2 Let $\Gamma: Y \rightrightarrows 2^{X}$ be a set-valued mapping and let $(\bar{y}, \bar{x}) \in \operatorname{gph} \Gamma$. Let $r_{\bar{x}}>0, r_{\bar{y}}>0$ and $M>0$. Then $\Gamma$ is said to be Lipchitz-like at $(\bar{y}, \bar{x})$ on $\mathbb{B}_{r_{\bar{y}}}(\bar{y}) \times \mathbb{B}_{r_{\bar{x}}}(\bar{x})$ with constant $M$ if the following inequqlity hold:

$$
e\left(\Gamma\left(y_{1}\right) \cap \mathbb{B}_{r_{\bar{x}}}(\bar{x}), \Gamma\left(y_{2}\right)\right) \leq M\left\|y_{1}-y_{2}\right\| \text { for any } y_{1}, y_{2} \in \mathbb{B}_{r_{\bar{y}}}(\bar{y}) .
$$

The following result establish the equivalence relation between metric regularity of a mapping $F$ at $(\bar{x}, \bar{y})$ and the Lipschitz-like continuity of the inverse $F^{-1}$ at $(\bar{y}, \bar{x})$, which is obtained from the idea in [19] [20].

Lemma 1 Let $F: X \rightrightarrows 2^{Y}$ be a set valued mapping and $\bar{y} \in F(\bar{x})$. Let $r_{\bar{x}}>0, r_{\bar{y}}>0$, then $F$ is metrically regular at $(\bar{x}, \bar{y})$ on $\mathbb{B}_{r_{\bar{x}}}(\bar{x}) \times \mathbb{B}_{r_{\bar{y}}}(\bar{y})$ if and only if its inverse $F^{-1}: Y \rightrightarrows 2^{X}$ is Lipschitz-like at $(\bar{y}, \bar{x})$ on $\mathbb{B}_{r_{\bar{y}}}(\bar{y}) \times \mathbb{B}_{r_{\bar{x}}}(\bar{x})$ with constant $\kappa>0$ such that

$$
e\left(F^{-1}(y) \cap \mathbb{B}_{r_{\bar{x}}}(\bar{x}), F^{-1}\left(y^{\prime}\right)\right) \leq \kappa\left\|y-y^{\prime}\right\| \text { for all } y, y^{\prime} \in \mathbb{B}_{r_{\bar{y}}}(\bar{y}) .
$$

We recall the following statement of Lyusternik-Graves theorem for metrically regular mapping from [21]. This theorem plays an important role in the theory of metric regularity and proves the stability of metric regularity of a generalized equation under perturbations. For its statement, we use that a set $C \subset X$ is locally closed at $z \in C$ if there exists $a>0$ such that the set $C \cap \mathbb{B}_{a}(z)$ is closed.

Lemma 2 Consider a mapping $F: X \rightrightarrows 2^{Y}$ and any $(\bar{x}, \bar{y}) \in \operatorname{gph} F$ at which $\operatorname{gph} F$ is locally closed. Let $F$ be metrically regular at $\bar{x}$ for $\bar{y}$ with constant $\kappa>0$. Consider also a function $g: X \rightarrow Y$ which is Lipschitz continuous at $\bar{x}$ with Lipschitz constant $\lambda$ such that $\lambda<\kappa^{-1}$. Then the mapping $g+F$ is metrically regular at $\bar{x}$ for $\bar{y}+g(\bar{x})$ with constant $\frac{\kappa}{1-\kappa \lambda}$.

We finished this section with the following lemma, which is known as Banach fixed point theorem proved in [22].

Lemma 3 Let $\Phi: X \rightrightarrows 2^{X}$ be a set-valued mapping. Let $\eta_{0} \in X, r \in(0, \infty)$ and $\alpha \in(0,1)$ be such that

$$
\operatorname{dist}\left(\eta_{0}, \Phi\left(\eta_{0}\right)\right)<r(1-\alpha)
$$

and

$$
e\left(\Phi\left(x_{1}\right) \cap \mathbb{B}_{r}\left(\eta_{0}\right), \Phi\left(x_{2}\right)\right) \leq \alpha\left\|x_{1}-x_{2}\right\| \quad \text { for any } x_{1}, x_{2} \in \mathbb{B}_{r}\left(\eta_{0}\right) .
$$

Then $\Phi$ has a fixed point in $\mathbb{B}_{r}\left(\eta_{0}\right)$, that is, there exists $x \in \mathbb{B}_{r}\left(\eta_{0}\right)$ such that $x \in \Phi(x)$. If $\Phi$ is additionally single-valued, then the fixed point of $\Phi$ in $\mathbb{B}_{r}\left(\eta_{0}\right)$ is unique.

\section{Convergence Analysis of GG-PPA}

In this section, we assume that $T: X \rightrightarrows 2^{Y}$ is a set-valued mapping with locally closed graph at $(\bar{x}, \bar{y}) \in \operatorname{gph} T$ such that $T$ is metrically regular at $(\bar{x}, \bar{y})$ with constant $\kappa>0$. Let $g: X \rightarrow Y$ be a (single-valued) function such that $g(0)=0$, which is Lipschitz continuous in a neighborhood $O$ of 0 with a Lipschitz constant $\lambda>0$. Let $x \in X$ and define a mapping $P_{x}$ by

$$
P_{x}(\cdot):=g(\cdot-x)+T(\cdot)
$$

Then we obtain the following equivalence

$$
z \in P_{x}^{-1}(y) \Leftrightarrow y \in g(z-x)+T(z) \text { for any } z \in X \text { and } y \in Y .
$$

In particular,

$$
\bar{x} \in P_{\bar{x}}^{-1}(\bar{y}) \text { for each }(\bar{x}, \bar{y}) \in \operatorname{gph} T .
$$


Let $(\bar{x}, \bar{y}) \in \operatorname{gph}(g+T)$ and let $r_{\bar{x}}>0, r_{\bar{y}}>0$. Since $g(\cdot-\bar{x})$ is Lipschitz continuous on $O+\bar{x}$, applying the Lyusternik-Graves theorem (see Lemma 2) we assume that the mapping $P_{\bar{x}}$ is metrically regular at $(\bar{x}, \bar{y})$ with constant $\frac{\kappa}{1-\kappa \lambda}$, that is, by Lemma 1 we have the following inequality

$$
e\left(P_{\bar{x}}^{-1}(y) \cap \mathbb{B}_{r_{\bar{x}}}(\bar{x}), P_{\bar{x}}^{-1}\left(y^{\prime}\right)\right) \leq \frac{\kappa}{1-\kappa \lambda}\left\|y-y^{\prime}\right\| \text { for all } y, y^{\prime} \in \mathbb{B}_{r_{\bar{y}}}(\bar{y}) .
$$

Write

$$
\bar{r}:=\min \left\{\frac{2 r_{\bar{y}}-r_{\bar{x}} \lambda}{2}, \frac{r_{\bar{x}}(1-3 \kappa \lambda)}{4 \kappa}\right\} .
$$

Then

$$
\bar{r}>0 \Leftrightarrow \lambda<\min \left\{\frac{2 r_{\bar{y}}}{r_{\bar{x}}}, \frac{1}{3 \kappa}\right\} .
$$

The following lemma plays an important role for convergence analysis of the GG-PPA, which is due to [23].

Lemma 4 Suppose that $P_{\bar{x}}(\cdot)$ is metrically regular at $(\bar{x}, \bar{y})$ on $\mathbb{B}_{r_{\bar{x}}}(\bar{x}) \times \mathbb{B}_{r_{\bar{y}}}(\bar{y})$ with constant $\frac{\kappa}{1-\kappa \lambda}$ such that (12) and (13) are satisfied. Let $x \in \mathbb{B}_{\frac{r_{\bar{x}}}{2}}(\bar{x})$ and $\mathbb{B}_{r_{\bar{x}}}(0) \subset O$. Then $P_{x}^{-1}(\cdot)$ is Lipschitz-like at $(\bar{y}, \bar{x})$ on $\mathbb{B}_{\bar{r}}(\bar{y}) \times \mathbb{B}_{\frac{r_{\bar{x}}}{2}}(\bar{x})$ with constant $\frac{\kappa}{1-3 \kappa \lambda}$, that is,

$$
e\left(P_{x}^{-1}\left(y_{1}\right) \cap \mathbb{B}_{\frac{r_{\bar{x}}}{2}}(\bar{x}), P_{x}^{-1}\left(y_{2}\right)\right) \leq \frac{\kappa}{1-3 \kappa \lambda}\left\|y_{1}-y_{2}\right\| \quad \text { for any } y_{1}, y_{2} \in \mathbb{B}_{\bar{r}}(\bar{y}) .
$$

For our convenience, we consider a sequence of functions $g_{k}: X \rightarrow Y$ with $g_{k}(0)=0$ which are Lipschitz continuous in a neighbourhood $O$ of 0 , the same for all $k$, with Lipschitz constants $\lambda_{k}$ satisfying

$$
\lambda:=\sup _{k} \lambda_{k}<\frac{1}{6 \kappa} .
$$

We rewrite the mapping $P_{x}(\cdot)$ in (8) by substituting $g_{k}$ instead of $g$ as follows:

$$
P_{x}^{k}(\cdot):=g_{k}(\cdot-x)+T(\cdot) \text { for each } k=0,1,2, \cdots .
$$

Since $\lambda \kappa<\frac{1}{6}<1$ by (14), then by Lyusternik-Graves theorem (see Lemma 2) and Lemma 1 we obtain that the mapping $P_{\bar{x}}^{k-1}(\cdot)$ is Lipschitz-like at $(\bar{y}, \bar{x})$ on $\mathbb{B}_{r_{\bar{y}}}(\bar{y}) \times \mathbb{B}_{r_{\bar{x}}}(\bar{x})$ with constant $\frac{\kappa}{1-\kappa \lambda}$ satisfying (11) and hence we have

$$
D^{k}(x)=\left\{d \in X: 0 \in P_{x}^{k}(x+d)\right\} .
$$

Furthermore, we define, for each $x \in X$, the mapping $Z_{x}: X \rightrightarrows Y$ by

$$
Z_{x}^{k}(\cdot):=g_{k}(\cdot-\bar{x})-g_{k}(\cdot-x),
$$

and the set-valued mapping $\Phi_{x}^{k}: X \rightrightarrows 2^{X}$ by

$$
\Phi_{x}^{k}(\cdot):=P_{\bar{x}}^{k-1}\left[Z_{x}^{k}(\cdot)\right] .
$$

Then 


$$
\begin{aligned}
& \left\|Z_{x}^{k}\left(x^{\prime}\right)-Z_{x}^{k}\left(x^{\prime \prime}\right)\right\| \\
& =\left\|g_{k}\left(x^{\prime}-\bar{x}\right)-g_{k}\left(x^{\prime}-x\right)-g_{k}\left(x^{\prime \prime}-\bar{x}\right)+g_{k}\left(x^{\prime \prime}-x\right)\right\| \\
& \leq\left\|g_{k}\left(x^{\prime}-\bar{x}\right)-g_{k}\left(x^{\prime \prime}-\bar{x}\right)\right\|+\left\|g_{k}\left(x^{\prime}-x\right)-g_{k}\left(x^{\prime \prime}-x\right)\right\| \text { for each } x^{\prime}, x^{\prime \prime} \in X .
\end{aligned}
$$

The main result of this study given as follows, which provides some sufficient conditions ensuring the convergence of the GG-PPA with initial point $x_{0}$.

Theorem 1 Suppose $\eta>1$ and that $P_{\bar{x}}^{k}(\cdot)$ is metrically regular at $(\bar{x}, \bar{y})$ on $\mathbb{B}_{r_{\bar{x}}}(\bar{x}) \times \mathbb{B}_{r_{\bar{y}}}(\bar{y})$ with constant $\frac{\kappa}{1-\kappa \lambda}$, and let $\bar{r}$ be defined in (12). Let $\mathbb{B}_{r_{\bar{x}}}(\bar{x}) \subset O$ and $\delta>0$ be such that

a) $\delta \leq \min \left\{\frac{r_{\bar{x}}}{4}, \frac{\bar{r}}{2 \lambda}, \frac{r_{\bar{y}}}{3 \lambda}, 1, \frac{1-\lambda \kappa}{6 \kappa \lambda}\right\}$,

b) $(\eta+3) \kappa \lambda \leq 1$,

c) $\|\bar{y}\|<\lambda \delta$.

Suppose that

$$
\lim _{x \rightarrow \bar{x}} \operatorname{dist}(\bar{y}, T(x))=0 .
$$

Then there exists some $\hat{\delta}>0$ such that any sequence $\left\{x_{k}\right\}$ generated by Algorithm 2 with initial point in $\mathbb{B}_{\hat{\delta}}(\bar{x})$ converges to a solution $x^{*}$ of $(1)$, that is, $x^{*}$ satisfies that $0 \in T\left(x^{*}\right)$.

Proof. Let

$$
t:=\frac{\eta \kappa \lambda}{1-3 \kappa \lambda}
$$

Then by assumption (b), (21) gives us

$$
t \leq 1 \text {. }
$$

Assumption (c) and (20) allow us to take $0<\hat{\delta} \leq \delta$ so that

$$
\operatorname{dist}\left(0, T\left(x_{0}\right)\right) \leq \lambda \delta \text { for each } x_{0} \in \mathbb{B}_{\hat{\delta}}(\bar{x}) .
$$

We will proceed by mathematical induction and show that Algorithm 2 generates at least one sequence and any sequence $\left\{x_{k}\right\}$ generated by Algorithm 2 satisfies the following assertions

$$
\left\|x_{k}-\bar{x}\right\| \leq 2 \delta
$$

and

$$
\left\|x_{k+1}-x_{k}\right\| \leq t^{k+1} \delta
$$

for each $k=0,1,2, \cdots$. Define

$$
r_{x}:=\frac{5 \kappa}{3(1-\lambda \kappa)}(\|\bar{y}\|+\lambda\|x-\bar{x}\|) \text { for each } x \in X .
$$

Since $\eta>1$, by assumption (b) and (c), we have

$$
r_{x} \leq \frac{5 \kappa \lambda}{1-\lambda \kappa} \delta \leq \frac{5}{\eta+2} \delta \leq 2 \delta \text { for each } x \in \mathbb{B}_{2 \delta}(\bar{x}) .
$$

It is trivial that (24) is true for $k=0$. For showing that (25) is true for $k=0$, we need to prove that $x_{1}$ exists, that is, $D^{0}\left(x_{0}\right) \neq \varnothing$. We will prove $D^{0}\left(x_{0}\right) \neq \varnothing$ by applying Lemma 3 to the mapping $\Phi_{x_{0}}^{0}$ with $\eta=\bar{x}$. Let us check that both assertions (6) and (7) of Lemma 3 are hold with $r:=r_{x_{0}}$ and $\alpha:=\frac{2}{5}$. Noting that 
$\bar{x} \in P_{\bar{x}}^{0^{-1}}(\bar{y}) \cap \mathbb{B}_{r_{x_{0}}}(\bar{x})$ by (10). Then by the mapping $\Phi_{x_{0}}^{0}$ in (18) and the definition of excess $e$, we have

$$
\begin{aligned}
\operatorname{dist}\left(\bar{x}, \Phi_{x_{0}}^{0}(\bar{x})\right) & \leq e\left(P_{\bar{x}}^{0-1}(\bar{y}) \cap \mathbb{B}_{r_{x_{0}}}(\bar{x}), \Phi_{x_{0}}^{0}(\bar{x})\right) \leq e\left(P_{\bar{x}}^{0-1}(\bar{y}) \cap \mathbb{B}_{2 \delta}(\bar{x}), \Phi_{x_{0}}^{0}(\bar{x})\right) \\
& \leq e\left(P_{\bar{x}}^{0-1}(\bar{y}) \cap \mathbb{B}_{r_{\bar{x}}}(\bar{x}), P_{\bar{x}}^{0-1}\left[Z_{x_{0}}^{0}(\bar{x})\right]\right)
\end{aligned}
$$

(noting that $\mathbb{B}_{r_{x_{0}}}(\bar{x}) \leq \mathbb{B}_{2 \delta}(\bar{x}) \subseteq \mathbb{B}_{r_{\bar{x}}}(\bar{x})$ ). Now, by the choice of $\lambda$, we have

$$
\begin{aligned}
\left\|Z_{x_{0}}^{0}(x)-\bar{y}\right\| & =\left\|g_{0}(x-\bar{x})-g_{0}\left(x-x_{0}\right)-\bar{y}\right\| \leq\left\|g_{0}(x-\bar{x})-g_{0}\left(x-x_{0}\right)\right\|+\|\bar{y}\| \\
& \leq \lambda_{0}\left\|x_{0}-\bar{x}\right\|+\|\bar{y}\| \leq \lambda\left\|x_{0}-\bar{x}\right\|+\|\bar{y}\| .
\end{aligned}
$$

Since $x_{0} \in \mathbb{B}_{\hat{\delta}}(\bar{x}) \subset \mathbb{B}_{\delta}(\bar{x})$, by the fact $3 \lambda \delta \leq r_{\bar{y}}$ in assumption (a) and $\|\bar{y}\| \leq \lambda \delta$ in assumption (c), for each $x \in \mathbb{B}_{\delta}(\bar{x}),(29)$ implies that

$$
\left\|Z_{x_{0}}^{0}(x)-\bar{y}\right\| \leq 2 \lambda \delta \leq r_{\bar{y}},
$$

that is, for each $x \in \mathbb{B}_{\delta}(\bar{x}), Z_{x_{0}}^{0}(x) \in \mathbb{B}_{r_{\bar{y}}}(\bar{y})$. In particular,

$$
\begin{aligned}
\left\|Z_{x_{0}}^{0}(\bar{x})-\bar{y}\right\| & =\left\|-g_{0}\left(\bar{x}-x_{0}\right)-\bar{y}\right\| \leq\left\|g_{0}(0)-g_{0}\left(\bar{x}-x_{0}\right)\right\|+\|\bar{y}\| \\
& \leq \lambda_{0}\left\|x_{0}-\bar{x}\right\|+\|\bar{y}\| \leq \lambda\left\|x_{0}-\bar{x}\right\|+\|\bar{y}\| \leq 2 \lambda \delta \leq r_{\bar{y}} .
\end{aligned}
$$

Hence by using (31) and Lemma 1 for Lipschitz-like property in (28), we have

$$
\begin{aligned}
\operatorname{dist}\left(\bar{x}, \Phi_{x_{0}}^{0}(\bar{x})\right) & \leq \frac{\kappa}{1-\kappa \lambda}\left\|\bar{y}-Z_{x_{0}}^{0}(\bar{x})\right\| \leq \frac{\kappa}{1-\kappa \lambda}\left(\|\bar{y}\|+\lambda\left\|x_{0}-\bar{x}\right\|\right) \\
& =\left(1-\frac{2}{5}\right) r_{x_{0}}=(1-\alpha) r .
\end{aligned}
$$

This shows that assertion (6) of Lemma 3 is satisfied. Now, we show that the assertion (7) of Lemma 3 is satisfied. Let $x^{\prime}, x^{\prime \prime} \in \mathbb{B}_{r_{x_{0}}}(\bar{x})$. Then by assumption (a) and (27), we have $x^{\prime}, x^{\prime \prime} \in \mathbb{B}_{r_{x_{0}}}(\bar{x}) \subseteq \mathbb{B}_{2 \delta}(\bar{x}) \subseteq \mathbb{B}_{r_{\bar{x}}}(\bar{x})$ and $Z_{x_{0}}^{0}\left(x^{\prime}\right), Z_{x_{0}}^{0}\left(x^{\prime \prime}\right) \in \mathbb{B}_{r_{\bar{y}}}(\bar{y})$ by (30). By assumed Lipschitz-like property of $P_{\bar{x}}^{0^{-1}}(\cdot)$, we have

$$
\begin{aligned}
e\left(\Phi_{x_{0}}^{0}\left(x^{\prime}\right) \cap \mathbb{B}_{r_{x_{0}}}(\bar{x}), \Phi_{x_{0}}^{0}\left(x^{\prime \prime}\right)\right) & \leq e\left(\Phi_{x_{0}}^{0}\left(x^{\prime}\right) \cap \mathbb{B}_{r_{\bar{x}}}(\bar{x}), \Phi_{x_{0}}^{0}\left(x^{\prime \prime}\right)\right) \\
& =e\left(P_{\bar{x}}^{0-1}\left[Z_{x_{0}}^{0}\left(x^{\prime}\right)\right] \cap \mathbb{B}_{r_{\bar{x}}}(\bar{x}), P_{\bar{x}}^{0-1}\left[Z_{x_{0}}^{0}\left(x^{\prime \prime}\right)\right]\right) \\
& \leq \frac{\kappa}{1-\kappa \lambda}\left\|Z_{x_{0}}^{0}\left(x^{\prime}\right)-Z_{x_{0}}^{0}\left(x^{\prime \prime}\right)\right\| .
\end{aligned}
$$

Applying (19) in (32), we obtain

$$
\begin{aligned}
& e\left(\Phi_{x_{0}}^{0}\left(x^{\prime}\right) \cap \mathbb{B}_{r_{x_{0}}}(\bar{x}), \Phi_{x_{0}}^{0}\left(x^{\prime \prime}\right)\right) \\
& \leq \frac{\kappa}{1-\kappa \lambda}\left(\left\|g_{0}\left(x^{\prime}-\bar{x}\right)-g_{0}\left(x^{\prime \prime}-\bar{x}\right)\right\|+\left\|g_{0}\left(x^{\prime}-x_{0}\right)-g_{0}\left(x^{\prime \prime}-x_{0}\right)\right\|\right) \\
& \leq \frac{2 \kappa \lambda_{0}}{1-\kappa \lambda}\left\|x^{\prime}-x^{\prime \prime}\right\| \leq \frac{2 \kappa \lambda}{1-\kappa \lambda}\left\|x^{\prime}-x^{\prime \prime}\right\| .
\end{aligned}
$$

Then by (14), (33) reduces to

$$
e\left(\Phi_{x_{0}}^{0}\left(x^{\prime}\right) \cap \mathbb{B}_{x_{x_{0}}}(\bar{x}), \Phi_{x_{0}}^{0}\left(x^{\prime \prime}\right)\right) \leq \frac{2 \kappa \lambda}{1-\kappa \lambda}\left\|x^{\prime}-x^{\prime \prime}\right\| \leq \frac{2}{5}\left\|x^{\prime}-x^{\prime \prime}\right\|=\alpha\left\|x^{\prime}-x^{\prime \prime}\right\| .
$$

This implies that the assertion (7) of Lemma 3 is also satisfied. Since both assertions (6) and (7) of Lemma 3 are fulfilled, we can deduce there exists a fixed point $\hat{x}_{1} \in \mathbb{B}_{r_{00}}(\bar{x})$ such that $\hat{x}_{1} \in \Phi_{x_{0}}^{0}\left(\hat{x}_{1}\right)$, which translates to $Z_{x_{0}}^{0}\left(\hat{x}_{1}\right) \in P_{\bar{x}}^{0}\left(\hat{x}_{1}\right)$, that is, $0 \in g_{0}\left(\hat{x}_{1}-x_{0}\right)+T\left(\hat{x}_{1}\right)$ and hence $D^{0}\left(x_{0}\right) \neq \varnothing$. 
Now, we show that (25) is hold for $k=0$.

Note that $\bar{r}>0$ by assumption (a). Then (13) is valid for (14). Since $P_{\bar{x}}^{k^{-1}}(\cdot)$ is Lipschitz-like at $(\bar{y}, \bar{x})$ on $\mathbb{B}_{r_{\bar{y}}}(\bar{y}) \times \mathbb{B}_{r_{\bar{x}}}(\bar{x})$, it follows from Lemma 4 that the mapping $P_{x}^{k^{-1}}(\cdot)$ is Lipschitz-like at $(\bar{y}, \bar{x})$ on $\mathbb{B}_{\bar{r}}(\bar{y}) \times \mathbb{B}_{\frac{r_{\bar{x}}}{2}}(\bar{x})$ with constant $\frac{\kappa}{1-3 \kappa \lambda}$ for each $x \in \mathbb{B}_{\frac{r_{\bar{x}}}{2}}(\bar{x})$. In particular, $P_{x_{0}}^{0^{-1}}(\cdot)$ is Lipschitz-like at $(\bar{y}, \bar{x})$ on $\mathbb{B}_{\bar{r}}(\bar{y}) \times \mathbb{B}_{\frac{r_{\bar{x}}}{2}}(\bar{x})$ with constant $\frac{\kappa}{1-3 \kappa \lambda}$ as $x_{0} \in \mathbb{B}_{\hat{\delta}}(\bar{x}) \subset \mathbb{B}_{\delta}(\bar{x}) \subset \mathbb{B}_{\frac{r_{\bar{x}}}{2}}(\bar{x})$ by assumption (a) and the choice of $\hat{\delta}$. Furthermore, assumptions (a) and (c) imply that

$$
\|\bar{y}\|<\lambda \delta \leq \bar{r} .
$$

It seems that $0 \in \mathbb{B}_{\bar{r}}(\bar{y})$. Then by Lemma 1 we can say that the mapping $P_{x_{0}}^{0}(\cdot)$ is metrically regular on $\mathbb{B}_{\frac{r_{\bar{x}}}{2}}(\bar{x})$ relative to $\mathbb{B}_{\bar{r}}(\bar{y})$ with constant $\frac{\kappa}{1-3 \kappa \lambda}$. Thus by applying Lemma 1, we have

$$
\operatorname{dist}\left(x_{0}, P_{x_{0}}^{0^{-1}}(0)\right) \leq \frac{\kappa}{1-3 \kappa \lambda} \operatorname{dist}\left(0, P_{x_{0}}^{0}\left(x_{0}\right)\right)
$$

and (23) implies that

$$
\operatorname{dist}\left(x_{0}, P_{x_{0}}^{0^{-1}}(0)\right) \leq \frac{\kappa}{1-3 \kappa \lambda} \operatorname{dist}\left(0, P_{x_{0}}^{0}\left(x_{0}\right)\right)=\frac{\lambda \kappa}{1-3 \kappa \lambda} \operatorname{dist}\left(0, T\left(x_{0}\right)\right) \leq \frac{\lambda \kappa}{1-3 \kappa \lambda} \delta .
$$

Then from (16) and using (36), we obtain that

$$
\operatorname{dist}\left(0, D^{0}\left(x_{0}\right)\right)=\operatorname{dist}\left(x_{0}, P_{x_{0}}^{0^{-1}}(0)\right) \leq \frac{\lambda \kappa}{1-3 \kappa \lambda} \delta .
$$

From Algorithm 2 and using (21) and (37), we obtain that

$$
\left\|x_{1}-x_{0}\right\|=\left\|d_{0}\right\| \leq \eta \operatorname{dist}\left(0, D^{0}\left(x_{0}\right)\right) \leq \frac{\eta \kappa \lambda}{1-3 \kappa \lambda} \delta<t \delta .
$$

This implies that (25) is hold for $k=0$.

Suppose that the points $x_{1}, \cdots, x_{n}$ have been obtained, and (24) and (25) are true for $k=0,1,2, \cdots, n-1$. We will show that there exists a point $x_{n+1}$ such that (24) and (25) also hold for $k=n$. Since (24) and (25) are true for each $k \leq n-1$, we have the following inequality

$$
\left\|x_{n}-\bar{x}\right\| \leq \sum_{i=0}^{n-1}\left\|d_{i}\right\|+\left\|x_{0}-\bar{x}\right\| \leq \delta \sum_{i=0}^{n-1} t^{i+1}+\delta \leq \frac{\delta t}{1-t}+\delta \leq 2 \delta .
$$

This reflects that (24) holds for $k=n$. Now with almost the same argument as we did for the case when $k=0$, we can find that the mapping $P_{x_{n}}^{n^{-1}}(\cdot)$ is also Lipschitz-like at $(\bar{y}, \bar{x})$ on $\mathbb{B}_{\bar{r}}(\bar{y}) \times \mathbb{B}_{\frac{r_{\bar{x}}}{2}}(\bar{x})$ with constant $\frac{\kappa}{1-3 \kappa \lambda}$. Then by applying again Algorithm 2, we have

$$
\begin{aligned}
\left\|x_{n+1}-x_{n}\right\| & =\left\|d_{n}\right\| \leq \eta \operatorname{dist}\left(0, D^{n}\left(x_{n}\right)\right)=\eta \operatorname{dist}\left(x_{n}, P_{x_{n}}^{n^{-1}}(0)\right) \\
& \leq \frac{\eta \kappa}{1-3 \kappa \lambda} \operatorname{dist}\left(0, P_{x_{n}}^{n}\left(x_{n}\right)\right)=\frac{\eta \kappa}{1-3 \kappa \lambda} \operatorname{dist}\left(0, T\left(x_{n}\right)\right) \\
& =\frac{\eta \kappa}{1-3 \kappa \lambda} \operatorname{dist}\left(0,-g_{n-1}\left(x_{n}-x_{n-1}\right)\right)=\frac{\eta \kappa}{1-3 \kappa \lambda}\left\|g_{n-1}(0)-g_{n-1}\left(x_{n}-x_{n-1}\right)\right\| \\
& \leq \frac{\eta \kappa \lambda_{n-1}}{1-3 \kappa \lambda}\left\|x_{n}-x_{n-1}\right\| \leq \frac{\eta \kappa \lambda}{1-3 \kappa \lambda}\left\|x_{n}-x_{n-1}\right\| \leq \frac{\eta \kappa \lambda}{1-3 \kappa \lambda} t^{n} \delta \leq t^{n+1} \delta .
\end{aligned}
$$

This shows that (25) holds for $k=n$. Therefore, the proof is completed. 
In the particular case, when $\bar{x}$ is a solution of (1), that is, $\bar{y}=0$, Theorem 1 is reduced to the following corollary, which gives the local convergence of the sequence generated by the GG-PPA defined by Algorithm 2.

Corollary 1 Suppose that $\eta>1$ and that $\bar{x}$ satisfies $0 \in T(\bar{x})$ and that $P_{\bar{x}}^{k}(\cdot)$ is metrically regular at $(\bar{x}, 0)$ with constant $\frac{\kappa}{1-\lambda \kappa}$. Let $\tilde{r}>0$ be such that $\mathbb{B}_{2 \tilde{r}}(\bar{x}) \subset O$ and suppose that

$$
\lim _{x \rightarrow \bar{x}} \operatorname{dist}(0, T(x))=0 .
$$

Then there exists some $\hat{\delta}>0$ such that any sequence $\left\{x_{k}\right\}$ generated by Algorithm 2 with initial point in $\mathbb{B}_{\hat{\delta}}(\bar{x})$ converges to a solution $x^{*}$ of $(1)$, that is, $x^{*}$ satisfies that $0 \in T\left(x^{*}\right)$.

Proof. Since $P_{\bar{x}}^{k}(\cdot)$ is metrically regular at $(\bar{x}, 0)$, there exist constants $r_{0}, \hat{r}_{\bar{x}}$ and $\frac{\kappa}{1-\kappa \lambda}$ such that $P_{\bar{x}}^{k}(\cdot)$ is metrically regular at $(\bar{x}, 0)$ on $\mathbb{B}_{\hat{r}_{\bar{x}}}(\bar{x}) \times \mathbb{B}_{r_{0}}(0)$ with constant $\frac{\kappa}{1-\kappa \lambda}$. Then, for each $0<r \leq \hat{r}_{\bar{x}}$, one has that

$$
\operatorname{dist}\left(x, P_{\bar{x}}^{k^{-1}}(y)\right) \leq \frac{\kappa}{1-\kappa \lambda} \operatorname{dist}\left(y, P_{\bar{x}}^{k}(x)\right) \text { for all } x \in \mathbb{B}_{r_{\bar{x}}}(\bar{x}), y \in \mathbb{B}_{r_{0}}(0) .
$$

Let $\sup _{k} \lambda_{k}:=\lambda \in(0,1)$ be such that $\kappa \lambda \leq \frac{1}{\eta+3}$. Choose $r_{\bar{x}} \in\left(0, \hat{r}_{\bar{x}}\right)$ such that $\frac{r_{\bar{x}}}{2} \leq \tilde{r}$ and $r_{0}-\frac{r_{\bar{x}} \lambda}{2}>0$. Then

$$
\bar{r}=\min \left\{\frac{2 r_{0}-r_{\bar{x}} \lambda}{2}, \frac{r_{\bar{x}}(1-3 \kappa \lambda)}{4 \kappa}\right\}>0,
$$

and

$$
\min \left\{\frac{r_{\bar{x}}}{4}, \frac{\bar{r}}{2 \lambda}, \frac{r_{0}}{3 \lambda}, \frac{1-\lambda \kappa}{6 \kappa \lambda}\right\}>0 .
$$

Thus we can choose $0<\delta \leq 1$ such that

$$
\delta \leq \min \left\{\frac{r_{\bar{x}}}{4}, \frac{\bar{r}}{2 \lambda}, \frac{r_{0}}{3 \lambda}, \frac{1-\lambda \kappa}{6 \kappa \lambda}\right\} .
$$

Now it is routine to check that inequalities (a)-(c) of Theorem 1 are hold. Thus Theorem 1 is applicable to complete the proof of the corollary.

\section{Numerical Experiment}

We will provide, in this section, a numerical example to validate the semilocal convergence results of GG-PPA.

Example 1 Let $X=Y=\mathbb{R}, x_{0}=0.2, \eta=2, \lambda=0.2$ and $\kappa=0.5$. Define a set-valued mapping $T$ on $\mathbb{R}$ by $T(x)=\{-2 x+1,1\}$. Consider a sequence of Lipschitz continuous function $g_{n}$ with $g_{n}(0)=0$, which is defined by $g_{n}(x)=\frac{1}{3} x$. Then Algorithm 2 generates a sequence which is converges to $x^{*}=0.5$.

It is obvious from the statement that $T$ is metrically regular at $(0.2,0.6) \in \operatorname{gph} T$ and $g_{n}$ is Lipschitz continuous on the neighborhood of 0 with Lipschitz constant $\sup _{k} \lambda_{k}:=\lambda=0.2$. Consider $T(x):=-2 x+1$. Then from (3), we have that

$$
\begin{aligned}
D^{k}\left(x_{k}\right) & =\left\{d_{k} \in \mathbb{R}: 0 \in g_{k}\left(d_{k}\right)+T\left(x_{k}+d_{k}\right)\right\} \\
& =\left\{d_{k} \in \mathbb{R}: d_{k}=\frac{3-6 x_{k}}{5}\right\} .
\end{aligned}
$$

On the other hand, if $D^{k}\left(x_{k}\right) \neq \varnothing$ we obtain that 


$$
0 \in g_{k}\left(x_{k+1}-x_{k}\right)+T\left(x_{k+1}\right) \Rightarrow x_{k+1}=\frac{3-x_{k}}{5} .
$$

Thus from (40), we obtain that

$$
\left\|d_{k}\right\| \leq \frac{\eta \kappa \lambda}{1-3 \kappa \lambda}\left\|d_{k-1}\right\|
$$

For the given values of $\eta, \lambda, \kappa$, we see that $\frac{\eta \kappa \lambda}{1-3 \kappa \lambda}=\frac{2}{7}<1$. Thus, this implies that the sequence generated by Algorithm 2 converges linearly. Then the following Table 1, obtained by using Mat lab program, indicates that the solution of the generalized equation is 0.5 when $k=10$.

Moreover, in the case when $T(x)=\{-2 x+1,1\}$, we can sketch the following Figure 1:

\section{Conclusions}

In this study, we have established semi-local and local convergence results for the general version of Gauss-type proximal point algorithm for solving generalized equation under the assumptions that $\eta>1$, a sequence of functions $g_{k}: X \rightarrow Y$ with $g_{k}(0)=0$ which is Lipschitz continuous in a neighbourhood $O$ of the origin

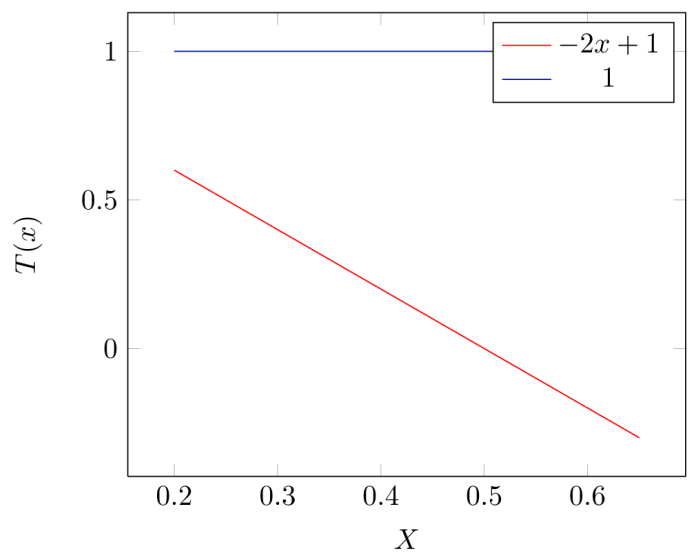

Figure 1. Graphical representation of $T(x)$.

Table 1. Finding a solution of generalized equation.

\begin{tabular}{cc}
$x$ & $T(x)$ \\
\hline 0.2000 & 0.6000 \\
0.5600 & -0.1200 \\
0.4880 & 0.0240 \\
0.5024 & -0.0048 \\
0.4995 & 0.0010 \\
0.5001 & -0.0002 \\
0.5000 & 0.0000 \\
0.5000 & -0.0000 \\
0.5000 & 0.0000 \\
0.5000 & -0.0000 \\
0.5000 & 0.0000 \\
\hline
\end{tabular}


and $T$ is metrically regular. Moreover, we have presented a numerical experiment to validate the semilocal convergence result for Algorithm 2. For the case where $\eta=1$, the question, whether the results are true for GG-PPA, is a little bit complicated. However, from the proof of the main theorem, one sees that all the results obtained in the present paper remain true provided that, for any $x \in \Omega \subseteq X$, the following implication holds:

$$
D(x) \neq \varnothing \Rightarrow \exists \bar{d} \in D(x) \text { such that }\|\bar{d}\|=\min _{d \in D(x)}\|d\| .
$$

To see the detail proof of the above implication, one can refer to [17].

\section{Acknowledgements}

We thank the editor and the referees for their comments. Research of this work is funded by the Ministry of Science and Technology, Bangladesh, grant No. 39.009.002.01.00.053.2014-2015/EAS-19. This support is greatly appreciated.

\section{References}

[1] Martinet, B. (1970) Régularisation d'inéquations variationnelles par approximations successives. Revue Française D'automatique, Informatique, Recherche Opérationnelle, 3, 154-158.

[2] Rockafellar, R.T. (1976) Monotone Operators and the Proximal Point Algorithm. SIAM Journal on Control and Optimization, 14, 877-898. http://dx.doi.org/10.1137/0314056

[3] Aragón Artacho, F.J., Dontchev, A.L. and Geoffroy, M.H. (2007) Convergence of the Proximal Point Method for Metrically Regular Mappings. ESAIM: Proceedings, 17, 1-8. http://dx.doi.org/10.1051/proc:071701

[4] Rashid, M.H., Wang, J.H. and Li., C. (2013) Convergence Analysis of Gauss-Type Proximal Point Method for Metrically Regular Mappings. Journal of Nonlinear and Convex Analysis, 14, 627-635.

[5] Auslender, A. and Teboulle, M. (2000) Lagrangian Duality and Related Multiplier Methods for Variational Inequality Problems. SIAM Journal on Optimization, 10, 1097-1115. http://dx.doi.org/10.1137/S1052623499352656

[6] Bauschke, H.H., Burke, J.V., Deutsch, F.R., Hundal, H.S. and Vanderwerff, J.D. (2005) A New Proximal Point Iteration that Converges Weakly But Not in Norm. Proceedings of the American Mathematical Society, 133, 1829-1835. http://dx.doi.org/10.1090/S0002-9939-05-07719-1

[7] Anh, P.N., Muu, L.D., Nguyen, V.H. and Strodiot, J.J. (2005) Using the Banach Contraction Principle to Implement the Proximal Point Method for Multivalued Monotone Variational Inequalities. Journal of Optimization Theory and Applications, 124, 285-306. http://dx.doi.org/10.1007/s10957-004-0926-0

[8] Yang, Z. and He, B. (2005) A Relaxed Approximate Proximal Point Algorithm. Annals of Operations Research, 133, 119-125. http://dx.doi.org/10.1007/s10479-004-5027-9

[9] Spingarn, J.E. (1982) Submonotone Mappings and the Proximal Point Algorithm. Numerical Functional Analysis and Optimization, 4, 123-150. http://dx.doi.org/10.1080/01630568208816109

[10] Iusem, A.N., Pennanen, T. and Svaiter, B.F. (2003) Inexact Variants of the Proximal Point Algorithm without Monotonicity. SIAM Journal on Optimization, 13, 1080-1097. http://dx.doi.org/10.1137/S1052623401399587

[11] Aragón Artacho, F.J. and Geoffroy, M.H. (2007) Uniformity and Inexact Version of a Proximal Point Method for Metrically Regular Mappings. Journal of Mathematical Analysis and Applications, 335, 168-183. http://dx.doi.org/10.1016/j.jmaa.2007.01.050

[12] Pennanen, T. (2002) Local Convergence of the Proximal Point Algorithm and Multiplier Methods without Monotonicity. Mathematics of Operations Research, 27, 170-191. http://dx.doi.org/10.1287/moor.27.1.170.331

[13] Li, C. and Ng, K.F. (2007) Majorizing Functions and Convergence of the Gauss-Newton Method for Convex Composite Optimization. SIAM Journal on Optimization, 18, 613-642. http://dx.doi.org/10.1137/06065622X

[14] Li, C., Zhang, W.H. and Jin, X.Q. (2004) Convergence and Uniqueness Properties of Gauss-Newton's Method. Computers \& Mathematics with Applications, 47, 1057-1067. http://dx.doi.org/10.1016/S0898-1221(04)90086-7

[15] He, J.S., Wang, J.H. and Li, C. (2007) Newton's Method for Underdetermined Systems of Equations under the Modified $\gamma$-Condition. Numerical Functional Analysis and Optimization, 28, 663-679. http://dx.doi.org/10.1080/01630560701348509

[16] Xu, X.B. and Li, C. (2008) Convergence Criterion of Newton's Method for Singular Systems with Constant Rank Derivatives. Journal of Mathematical Analysis and Applications, 345, 689-701. http://dx.doi.org/10.1016/j.jmaa.2008.04.009 
[17] Rashid, M.H., Yu, S.H., Li, C. and Wu, S.Y. (2013) Convergence Analysis of the Gauss-Newton-Type Method for Lipschitz-Like Mappings. Journal of Optimization Theory and Applications, 158, 216-233. http://dx.doi.org/10.1007/s10957-012-0206-3

[18] Aubin, J.P. and Frankowska, H. (1990) Set-Valued Analysis. Birkhäuser, Boston.

[19] Rockafellar, R.T. and Wets, R.J-B. (1997) Variational Analysis. Springer-Verlag, Berlin.

[20] Ioffe, A.D. (2000) Metric Regularity and Subdifferential Calculus. Russian Mathematical Surveys, 55, 501-558. http://dx.doi.org/10.1070/RM2000v055n03ABEH000292

[21] Dontchev, A.L., Lewis, A.S. and Rockafellar, R.T. (2002) The Radius of Metric Regularity. Transactions of the AMS, 355, 493-517. http://dx.doi.org/10.1090/S0002-9947-02-03088-X

[22] Dontchev, A.L. and Hager, W.W. (1994) An Inverse Mapping Theorem for Set-Valued Maps. Proceedings of the American Mathematical Society, 121, 481-498. http://dx.doi.org/10.1090/S0002-9939-1994-1215027-7

[23] Rashid, M.H. (2014) Convergence Analysis of Gauss-Type Proximal Point Method for Variational Inequalities. Open Science Journal of Mathematics and Application, 2, 5-14.

\section{Submit or recommend next manuscript to SCIRP and we will provide best service for you:}

Accepting pre-submission inquiries through Email, Facebook, Linkedin, Twitter, etc A wide selection of journals (inclusive of 9 subjects, more than 200 journals)

Providing a 24-hour high-quality service

User-friendly online submission system

Fair and swift peer-review system

Efficient typesetting and proofreading procedure

Display of the result of downloads and visits, as well as the number of cited articles

Maximum dissemination of your research work

Submit your manuscript at: http://papersubmission.scirp.org/ 